\title{
Hemolytic Activity of Trichomonas vaginalis and Tritrichomonas foetus
}

\section{Geraldo A De Carli/ ${ }^{+}$, Philippe Brasseur*, Ana C da Silva, Aline Wendorff, Marilise Rott}

\author{
Departamento de Análises, Faculdade de Farmácia, Universidade Federal do Rio Grande do Sul, \\ 90610-000 Porto Alegre, RS, Brasil *Laboratoire de Parasitologie, Hôpital Charles Nicolle, 76031 \\ Rouen Cédex, France
}

The hemolytic activity of live isolates and clones of Trichomonas vaginalis and Tritrichomonas foetus was investigated. The isolates were tested against human erythrocytes. No hemolytic activity was detected by the isolates of $\mathrm{T}$. foetus. Whereas the isolates of $\mathrm{T}$. vaginalis lysed erythrocytes from all human blood groups. No hemolysin released by the parasites could be detected. Our preliminary results suggest that hemolysis depend on the susceptibility of red cell membranes to destabilization and the intervention of cell surface receptors as a mechanism of the hemolytic activity. The mechanism could be subject to strain-species-genera specific variation of trichomonads. The hemolytic activity of T. vaginalis is not due to a hemolysin or to a product of its metabolism. Pretreatment of trichomonads with concanavalin A reduced levels of hemolysis by $40 \%$.

Key words: Trichomonas vaginalis - Tritrichomonas foetus - hemolytic activity - isolates - clones

Trichomonas vaginalis is a common cause of the infection of the female genital tract and trichomoniasis is recognized as a major sexually transmitted disease, while the Tritrichomonas foetus is responsible for the genital bovine urogenital trichomoniasis. However, the mechanisms of the pathogenicity of $T$. vaginalis and $T$. foetus have not yet been well defined. The pathogenicity of isolates of $T$. vaginalis and $T$. foetus have been previously reported, using mouse inoculation (Schnitzer et al. 1950, Bogovsky \& Teras 1958, Honigberg 1961, Reardon et al. 1961, Frost \& Honigberg 1962, Gobert et al. 1971, Dohnalová \& Kulda 1975), growth characteristics (Kulda \& Honigberg 1969, Kulda et al. 1970, Winston 1974), and also cytopathic effect assay (Brasseur \& Savel 1982, Alderete \& Pearlman 1984, Rasmussen et al. 1986, Roussel et al. 1991). Various systems have been developed to determine the hemolytic activity of T. vaginalis (Grys \& Hernik 1973, 1974, Krieger et al. 1983, De Carli et al. 1989, Dailey et al. 1990, Potamianos et al. 1992), however, up to the present, the hemolytic activity of $T$. foetus has not been studied. The aim of this study is to determine a hemolytic activity of $T$. foetus and of $T$. vaginalis.

This investigation was supported by Research Grants 40.106.92.6 and 93.2457.6, from CNPq and FAPERGS ${ }^{+}$Corresponding author Received 2 May 1995

Accepted 27 June 1995

\section{MATERIALS AND METHODS}

Organisms - All strains of T. vaginalis (VG, $\mathrm{GB}$, BoA, and Pc strains) studied were isolated from women with symptomatic vaginitis attending the Venereal Disease Department of the Charles Nicolle Hospital, Rouen, France. Four $T$. foetus strains used in this study (K, KV1, 5022, and PAL strains) were obtained from Prof. Wanderley de Souza (Institute of Biophysics, Federal University of Rio de Janeiro) and Helio Guida, DVM (EMBRAPA, Seropédica, RJ). The trichomonads were cultured axenically in vitro in trypticase-yeast extract-maltose (TYM) medium (Diamond 1957), supplemented with $10 \%$ heat inactivated cold horse serum at $37^{\circ} \mathrm{C}$. The $\mathrm{pH}$ of TYM medium was adjusted to 7.0-7.2 for T. foetus and 6.0 for $T$. vaginalis. Isolates were subcultured every $48 \mathrm{hr}$ in TYM medium. The strains were stored in liquid nitrogen $\left(-196^{\circ} \mathrm{C}\right)$ with $5 \%$ of dimethyl sulfoxide (DMSO) (Warton \& Honigberg 1980). The trichomonads in the logarithmic phase of growth and subcultured every $48 \mathrm{hr}$ exhibited more than $95 \%$ mobility and normal morphology. The protozoa were counted with a hemocytometer and adjusted to a concentration of $1 \times 10^{6}$ living organisms per $\mathrm{ml}$ in TYM medium. Isolation of $T$. vaginalis clones followed the method recommended by Linstead (1989).

Erythrocytes - Fresh human blood was obtained at the City Emergency Hospital (HPS) blood center and also from volunteer donors. The blood was taken in an equal volume of Alsever's solution (dextrose $20.5 \mathrm{~g}$, sodium citrate $8.0 \mathrm{~g}$, citric acid 
$0.55 \mathrm{~g}$, sodium chloride $4.2 \mathrm{~g}$, distilled water to 1 liter).

The erythrocytes were harvested and washed three times by centrifugation ( $250 \mathrm{x} \mathrm{g}$ for $10 \mathrm{~min})$ in equal volume of Hank's balanced salt solution (HBSS) (Bio-Merieux, France). The supernatant was discarded. Each experiment was performed using fresh erythrocytes from all human blood groups. Whole human blood samples were previously examined and determined to be hepatitis B antigen (HBsAg) negative and human immunodeficiency virus (HIV-antibody) negative. The erythrocytes were stored at $4^{\circ} \mathrm{C}$.

Hemolysis assay - The parasites were harvested from a $24 \mathrm{hr}$ culture in TYM medium at $37^{\circ} \mathrm{C}$ and washed three times in HBSS by centrifugation (750 $\mathrm{x} \mathrm{g}$ for $20 \mathrm{~min}$ ). A volume of $50 \mu \mathrm{l}$ of washed fresh undiluted erythrocytes was mixed with 2.5 $\mathrm{ml}$ of HBSS containing a total of $1 \times 10^{6}$ trophozoites of T. vaginalis or T. foetus (Krieger et al. 1983 ) originated from a $24 \mathrm{hr}$ culture in TYM medium. After $18 \mathrm{hr}$ of incubation at $37^{\circ} \mathrm{C}$ the mixture was centrifugated $(250 \mathrm{x}$ g for $10 \mathrm{~min}$ ). Absorbance of the supernatant was measured at $540 \mathrm{~nm}$ (De Carli et al. 1989) with a spectrometer (Schimadzen UV 160) and was compared with a standard curve obtained by osmotic lysis of the erythrocytes of each species. Control tubes were included in all assays and the spontaneous hemolysis was also controlled. The results were expressed as percentage of total hemolysis (100\%). The mean and the standard error of the hemolytic activity of every trichomonad species with the different erythrocytes were calculated after performing the assay at least 12 times in triplicate.

Lectins - Concanavalin A (Con A), from Sigma Chemical Co., St. Louis, MO, USA, was used in the concentration of $10 \mu \mathrm{g} / \mathrm{ml}$ diluted in phosphate buffered saline (PBS) $0.1 \mathrm{M} \mathrm{pH}$ 7.2. Equal volumes of trichomonads and Con A were incubated at $25^{\circ} \mathrm{C}$ under constant agitation. After $1 \mathrm{hr}$ of incubation the flagellates were washed three times in $0.1 \mathrm{M}$ PBS and harvested by centrifugation at $750 \mathrm{x} g$ for 20 min (Warton \& Honigberg 1980, Warton \& Papadimitriou 1984). This experiment was done with human blood group type O.

\section{RESULTS}

T. foetus (K, KV1, 5022, and PAL strains) did not present any hemolytic activity against all human blood groups (Table I). The parasites maintained their mobility and did not show any morphologic abnormality in the tests without hemolysis. Also, no hemolysis was detected when T. foetus strains were tested against rabbit, rat and chicken erythrocytes.

T. vaginalis $\mathrm{VG}, \mathrm{Gb}$, Boa, and Pc strains hemo-

\section{TABLE I}

Hemolytic activity of four isolates and two clones of Trichomonas vaginalis and four isolates of

Tritrichomonas foetus against human erythrocytes

\begin{tabular}{lccccc}
\hline & \multirow{2}{*}{$\begin{array}{c}\text { Number } \\
\text { of }\end{array}$} & \multicolumn{4}{c}{$\begin{array}{c}\text { Hemolysis }(\%)^{a} \\
\text { Human erythrocytes }\end{array}$} \\
\cline { 3 - 6 } Isolates & assays & $\mathrm{A}$ & $\mathrm{B}$ & $\mathrm{AB}$ & $\mathrm{O}$ \\
\hline VG & 12 & $68 \pm 0.2$ & $52 \pm 0.5$ & $60 \pm 0.6$ & $67 \pm 0.3$ \\
GB & 12 & $92 \pm 0.2$ & $75 \pm 0.2$ & $76 \pm 0.2$ & $96 \pm 0.2$ \\
Boa & 12 & $76 \pm 0.3$ & $57 \pm 0.3$ & $76 \pm 0.2$ & $76 \pm 0.7$ \\
Pc & 12 & $70 \pm 1.0$ & $52 \pm 1.1$ & $65 \pm 1.0$ & $66 \pm 0.6$ \\
C1 & 12 & $73 \pm 0.2$ & $56 \pm 0.2$ & $59 \pm 0.1$ & $79 \pm 0.2$ \\
C2 & 12 & $65 \pm 0.2$ & $59 \pm 0.3$ & $56 \pm 0.2$ & $72 \pm 0.3$ \\
K & 12 & $\mathrm{NH}$ & $\mathrm{NH}$ & $\mathrm{NH}$ & $\mathrm{NH}$ \\
KV1 & 12 & $\mathrm{NH}$ & $\mathrm{NH}$ & $\mathrm{NH}$ & $\mathrm{NH}$ \\
5022 & 12 & $\mathrm{NH}$ & $\mathrm{NH}$ & $\mathrm{NH}$ & $\mathrm{NH}$ \\
PAL & 12 & $\mathrm{NH}$ & $\mathrm{NH}$ & $\mathrm{NH}$ & $\mathrm{NH}$ \\
\hline
\end{tabular}

$a$ :the values represent the mean \pm the standard error of triplicate samples. $\mathrm{NH}=$ no hemolysis.

lyzed all human blood groups (Table I), as well as rabbit, rat and chicken. All isolates tested presented a hemolytic activity from 52 to $96 \%$. Hemolytic activity was maintained after a serial transfer in axenic culture for six months. The hemolytic activity varies according to donors origin of erythrocytes. No hemolysin released by the parasites could be identified.

Hemolysis did not occur with trichomonads culture supernatants from 24 and $48 \mathrm{hr}$ kept at $37^{\circ} \mathrm{C}$ (Table II). Hemolytic activity was not observed with the hemolysis supernatant from $18 \mathrm{hr}$, and neither with sonicated extracts of trichomonads, and nor with previously killed organisms (Table II).

The hemolytic activity was reduced in $40 \%$ by the pre-treatment of $T$. vaginalis with Con A (Table III).

\section{TABLE II}

The effect of Trichomonas vaginalis culture supernatants from 24 and $48 \mathrm{hr}$, hemolysis supernatant from $18 \mathrm{hr}$, parasites sonicated extracts and killed organisms against human erythrocytes

\begin{tabular}{lccccc}
\hline \multicolumn{1}{c}{ Number } & \multicolumn{4}{c}{ Hemolysis (\%) } \\
\multicolumn{1}{c}{ of } & \multicolumn{2}{c}{ Human erythrocytes } \\
& assays & A & B & AB & O \\
\hline Culture supernatants & 12 & NH & NH & NH & NH \\
Hemolysis supernatant & 12 & NH & NH & NH & NH \\
Sonicated extracts & 12 & NH & NH & NH & NH \\
Killed organisms & 12 & NH & NH & NH & NH \\
\hline
\end{tabular}

$a$ : culture supernatants from $1 \times 10^{6}$ trichomonads, hemolysis supernatant, parasites sonicated extracts and killed organisms were substituted for the trichomonads in the hemolysis assay. $\mathrm{NH}=$ no hemolysis. 


\section{TABLE III}

Hemolytic activity of pretreatment strains of Trichomonas vaginalis with Concanavalin A against human erythrocytes of group type $\mathrm{O}$

\begin{tabular}{lccccc}
\hline & Number & \multicolumn{4}{c}{ Hemolysis (\%) } \\
of & Isolates & \\
Treatment & assays & VG & GB & Boa & Pc \\
\hline None & 12 & $67 \pm 0.3$ & $96 \pm 0.2$ & $76 \pm 0.1$ & $66 \pm 0.6$ \\
Con A & 12 & $26 \pm 0.4$ & $38 \pm 0.1$ & $29 \pm 0.2$ & $26 \pm 0.3$
\end{tabular}

$a$ : the hemolytic activity was determined as described in the materials and methods section. The values represent the mean \pm the standard error of triplicate samples.

\section{DISCUSSION}

The hemolytic activity of some parasite protozoa was shown, particularly in Trypanosoma congolense (Tizard et al. 1977), Trypanosoma brucei (Tizard et al. 1978), Entamoeba histolytica (López-Revilla \& Said-Fernández 1980) and T. vaginalis (Grys \& Hernik, 1973, 1974, Krieger et al. 1983, Brasseur \& Savel 1982, De Carli et al. 1989, Dailey et al. 1990, Potamianos et al. 1992), but probably the hemolytic activity does not follow the same mechanism in these different parasite species.

It was reported that the hemolytic activity of $T$. congolense is connected with the liberation of fatty acids by the action of a phospholipase $\mathrm{A}$ on the endogenous phosphatidyl choline (Tizard \& Holmes 1976). The mechanism of this activity in $T$. vaginalis and $E$. histolytica has not yet been established. The strongest hemolytic potency in E. histolytica was observed in the most virulent strains (López-Revilla \& Said-Fernández 1980). However, there is no correlation between this activity and an enterotoxin isolated from this amoeba (Lushbaugh et al. 1979).

Bacterial hemolysins have been reported (Freer \& Arbuthnott 1976) in staphylococci, streptococci, clostridia, vibrios, and aeromonas and have been confirmed to correlate with virulence in many species.

The hemolysis depends on the susceptibility of red blood cells membranes to destabilization (López-Revilla \& Said-Fernández 1980).

Differences exist in different individuals of the same animal species in the susceptibility to a certain hemolysin (Cooper et al. 1966).

No enterotoxin was ever made evident (Brasseur \& Savel 1982), and no hemolytic activity was observed with culture supernatants. Nevertheless, our results are not in opposition with the finding that hemolytic activity is dependent upon adherence of red blood cells to the surface of T. vaginalis (Potamianos et al. 1992).
Our preliminary results suggest that the hemolytic activity is not due to the hemolysin released by T. vaginalis or to a product of its metabolism. It is possible that the hemolytic activity remains in the dependence of parasitic and erythrocytic cell surface receptors which probably carry mannose, because this activity is strongly reduced by pre-treatment of the parasites with Con A. These data suggest the intervention of the cell surface receptors as a mechanism of the hemolytic activity. This mechanism could be subject to strain-species-genera specific variation of trichomonads.

A complete study of the activity of lectins and saccharides will allow the identification of specific receptors implicated in this activity.

However, $T$. foetus isolates do not present any hemolytic activity against rabbit, rat, chicken, and erythrocytes from all human blood groups. This is the most important point which requires further studies. Probably many mechanisms determine the pathogenic potential and hemolytic activity of the trichomonad trophozoites. It is essential that modern molecular characterization studies be conducted in conjunction with biological studies to determine the significance of hemolytic activity of $T$. vaginalis.

The recent isolation of intact $T$. vaginalis DNA (Riley \& Krieger 1992) indicates the feasibility of further investigation and differentiation of these trichomonads using genetic engineering techniques.

\section{REFERENCES}

Alderete JF, Pearlman E 1984. Pathogenic Trichomonas vaginalis cytotoxity to cell culture monolayers. $B J$ Vener Dis 60: 99-105.

Bogovky PA, Teras J 1958. Pathologic-anatomical changes in white mice in intra-peritoneal infection by culture of Trichomonas vaginalis. Med Parazitol Parazit Bolenzi 27: 194-199.

Brasseur P, Savel J 1982. Evaluation de la virulance des souches de Trichomonas vaginalis par l'étude de l'effet cytopathogène sur culture de cellules. $C R$ Soc Biol 176: 849-860.

Cooper LZ, Madoff MA, Weintein L 1966. Heat stability and species range of purified staphylococcal $\alpha$ toxin. J Bacteriol 91: 1606-1692.

Dailey DC, Chang T, Alderete JF 1990. Characterization of Trichomonas vaginalis haemolysis. Parasitology 101: 171-175.

Diamond LS 1957 The establishment of various Trichomonas of animals and man in axenic cultures. J Parasitol 43: 488-490.

De Carli GA, Brasseur P, Savel J 1989. Activité hemolitique de differentes souches et clones de $T$. vaginalis. Bull Soc Fr Parasitol 7: 13-16.

Dohnalová M, Kulda J 1975. Pathogenicity of Tritrichomonas foetus for the laboratory mouse. $J$ Parasitol 22: 61A. 
Freer JH, Arbuthnott JP 1976. Biochemical and morphologic alterations of membranes by bacterial toxins, p. 103-193. In AW Bernheiner, Mechanisms in bacterial toxicology. John Wiley \& Sons, Inc., New York, NY.

Frost J, Honigberg BM 1962. Comparative pathogenicity of Trichomonas vaginalis and Trichomonas gallinae for mice. II. Histopathology and subcutaneous lesions. J Parasitol 48: 898-918.

Gobert JG, Truchet H, Savel J 1971. Etude de l'endoparasitisme expérimental chez la souris de Trichomonas vaginalis. IV Etude histochimique des lésions des animaux parasites. Ann Parasit Hum Comp 5: 511-522.

Grys E, Hernik A 1973. Hemolysis of human and rabbit erythrocytes by T. vaginalis. Wiad Parazytol 19: 399-400.

Grys E, Hernik A 1974. Haemolysis of erythrocytes by Trichomonas vaginalis. Pol Tyg Lek 29: 267-269.

Honigberg BM 1961. Comparative pathogenicity of Trichomonas vaginalis and Trichomonas gallinae to mice. I. Gross pathology, quantitative evaluation of virulence and same factors affecting pathogenicity. J Parasitol 47: 545-571.

Kulda J, Honigberg BM 1969. Behavior and pathogenicity of Tritrichomonas foetus in chick liver cell cultures. J Parasitol 16: 479-495.

Kulda J, Honigberg BM, Frost JK, Hollander DH 1970. Pathogenicity of Trichomonas vaginalis. Amer $J$ Obst Gynecol 108: 908-918.

Krieger JN, Poisson MA, Rein MF 1983. Beta-hemolytic activity of Trichomonas vaginalis correlates with virulence. Infect Immun 41: 1291-1295.

Linstead D 1989. Cultivation of trichomonads parasitic in humans, p. 91-111. In BM Honigberg. Trichomonads parasitic in humans. Springer-Verlag, New York. NY.

López-Revilla R, Said-Fernández S 1980. Cytopathogenicity of Entamoeba histolytica hemolytic activity of trophozoite homogenates. Am J Trop Med Hyg 29: 200-212.

Lushbaugh WB, Kairalla AB, Cantey JR, Hofbauer AF, Pittmann FF 1979. Isolation of a cytotoxin enterotoxin from Entamoeba histolytica. J Infec Dis 139: 9-7.
Potamianos S, Mason PR, Read JS, Chikungauwo S 1992. Lysis of erythrocytes by Trichomonas vaginalis. Biosc Rep 12: 387-395.

Rasmussen SE, Nielsen MH, Lind I, Rhodes JM 1986. Morphological studies of the cytotoxity of Trichomonas vaginalis to normal human vaginal epithelial cells in vitro. Genitourinary Med 62: 240246.

Reardon LV, Ashburn LL, Jacob L 1961. Differences in strains of Trichomonas vaginalis as revealed by intra-peritoneal injections into mice. J Parasitol 47: 527-532.

Riley DE, Krieger JN 1992. Rapid and practical DNA isolation from Trichomonas vaginalis and other nuclease-rich protozoa. Mol Biochem Parasitol 51: 161-164.

Roussel F, De Carli G, Brasseur PH 1991. A cytopathic effect of Trichomonas vaginalis probably mediated by a mannose/n-acetyl-glucosamine binding lectin. Int J Parasitol 21: 941-944.

Schnitzer D, Kelly R, Leiwant B 1950. Experimental studies on trichomoniasis: I. The pathogenicity of trichomonads species for mice. J Parasitol 36: 343348.

Tizard IR, Holmes WL 1976. The generation of toxic activity from Trypanosoma congolense. Experientia 32: $1533-1534$

Tizard IR, Holmes WL, York DA, Mellors A 1977. The generation and identification of the hemolysin of Trypanosoma congolense. Experientia 33: 901-902.

Tizard IR, Sheppard J, Nielsen K 1978. The characterization of a second class of hemolysins from Trypanosoma brucei. Trans $R$ Soc Trop Med Hyg 72: 198-200.

Warton A, Honigberg BM 1980. Lectin analysis of surface saccharides in two Trichomonas vaginalis strains differing in pathogenicity. J Parasitol 27: 410-419.

Warton A, Papadimitriou JM 1984. Binding of concanavalin $\mathrm{A}$ and wheat germ agglutinin by murine peritoneal macrophages: ultrastructural and cytophotometric studies. Histochem J 16: 1193-1206.

Winston RML 1974. The relations between size and pathogenicity of Trichomonas vaginalis. J Obstet Gynaecol Br Commonw 81: 399-404. 